\title{
Direct observation of the velocity contribution to friction in monolayer-coated micromachines
}

Sameer S. Shroffa and Maarten P. de Boera

Keywords: friction, MEMS, monolayers, micromachines

\section{Graphical abstract}

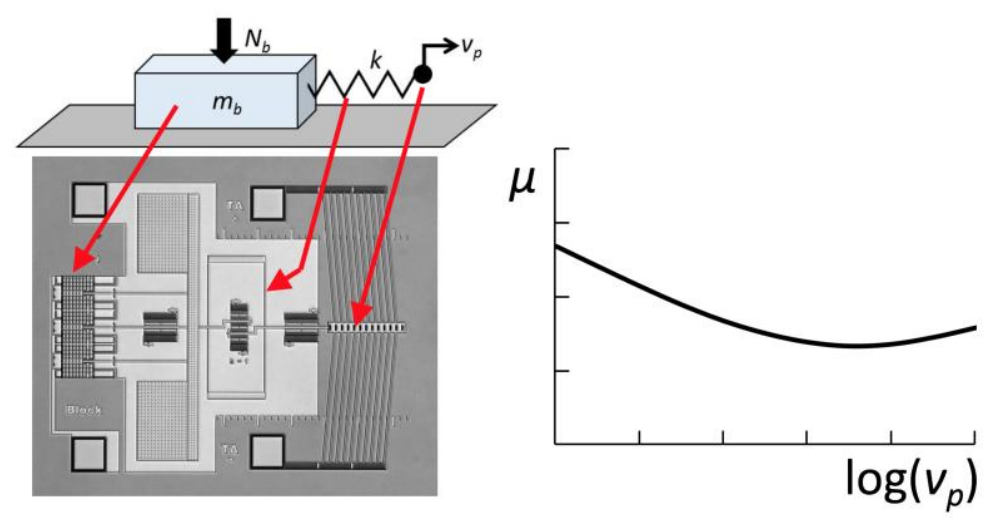

\begin{abstract}
Using a newly developed in-situ platform combined with high-speed imaging up to 200,000 frames per second, we measure friction versus velocity in a micromachined interface from 0.1 to $3000 \mu \mathrm{m} / \mathrm{s}$. The steadystate friction coefficient (friction at constant velocity) of a boundary lubricant-coated interface decreases up to $300 \mu \mathrm{m} / \mathrm{s}$, then increases. The friction increase is confirmed in an independent test in which velocity is increased instantaneously. Previous single asperity molecular dynamics simulations of monolayer boundary lubricants indicate that friction is independent of velocity, while experiments and simulations of periodic potentials indicate a monotonically increasing trend. We directly bridge spatial and temporal scales using rate-state constitutive laws, and attribute the decrease in friction to the decrease in asperity lifetime (a state effect), and the increase to the emerging dominance of interfacial viscosity (a rate effect).
\end{abstract}

\section{Introduction}

Friction derives from the shear strength between contacting points of two bodies. Surface roughness, spring constant, time, and velocity all influence friction, and determine whether stick-slip or steady sliding occurs. When two surfaces are pressed together, due to surface roughness they make contact only at high points known as asperities. To first order, the increase in friction force with normal load is due to a proportional increase in real contact area, explaining the usefulness of the friction coefficient [1]. However, the shear strength of the contacts at constant normal load can change with time or velocity. These effects have been treated by rate-state theory $[\underline{2}, \underline{3}]$. In that context, the asperity lifetime is inversely proportional to the relative velocity between the upper and lower counterfaces. 
Friction is a critical property in micrometer and nanometer-scale devices as their surface-to-volume ratio is high [4]. In microelectromechanical systems (MEMS), understanding the behavior of surfaces in sliding contact remains one of the most challenging issues due to unpredictable sliding behavior and wear []ㅡ. While static aging is known to occur []], the effect of block velocity under steady sliding conditions, $v$, has not previously been characterized in a representative micromachined multi-contact interface. In this paper, we explore the effect of $v$ on friction in a micromachined interface under conditions of low wear.

Polycrystalline silicon surfaces in MEMS consist of multiple nanometer-scale asperities and are coated by organic alkyl monolayers, which eliminate capillary adhesion and reduce friction forces by a factor of two or more [4]. The velocity dependence of friction in single asperity monolayer-coated systems has been investigated using modeling and experimental methods. In molecular dynamics (MD) simulations with monolayer coatings, it is reported that there is no rate effect [7]. This is because the simulations must use high velocities $(0.2 \mathrm{~m} / \mathrm{s}$ to $100 \mathrm{~m} / \mathrm{s}$ ) to be computationally practical, much higher than those in MEMS (0 to $\sim 1000 \mu \mathrm{m} / \mathrm{s}$ ). At such high velocities, there is insufficient time for thermal effects to play a significant role, and the creation of gauche defects in the alkyl chains is the main energy dissipation mechanism.

Researchers have used experimental scanning probe microscopy (SPM) methods to examine the velocity effect with organic monolayers coating both the nanometer-scale tip and the substrate. Here, $\mu$ is found to increase with $v$ from 0.3 to $100 \mu \mathrm{m} / \mathrm{s}$ [ []], and from 0.004 to $0.2 \mu \mathrm{m} / \mathrm{s}$ [9] ]. Both works invoked a viscoelastic spring-damper model, which predicts that $\mu$ would eventually decrease at higher velocities.

The Prandtl-Tomlinson model [10] also applies at velocities similar to those in MEMS. This model considers a point mass experiencing a periodic potential, analogous to a sharp tip sliding over a crystalline substrate. In the absence of a coating, the coefficient of friction, $\mu$, is found to increase with $v$, as the influence of thermal vibrations diminishes $[\underline{11}, \underline{12}]$. This prediction [11] has been validated experimentally [12]. In this case, MD simulations for a gold on platinum (uncoated) system were able to capture the monotonically increasing effect of stick-slip friction with velocity [13]. However, the model depends on lattice periodicity, while monolayer-coated surfaces are unlikely to present an ordered interface [14]].

In summary, single asperity models and experiments reveal friction increasing or remaining constant as velocity increases. The viscoelastic spring-damper model, while not verified experimentally, predicts a friction increase then decrease with $v$.

MEMS interfaces possess several key characteristics that are not well captured in such simulations and experiments. First, due to high asperity curvature (radius of curvature $\sim 25-100 \mathrm{~nm}$ ), the monolayer density and structure on the asperities of a micromachined surface is likely to be different than on the smooth single crystal surfaces often studied using MD [15]. Second, both surfaces are coated. Many SPM experiments [1619] use a single coated surface. Third, and perhaps most important, the multi-asperity surfaces possess a 2-8 $\mathrm{nm}$ root mean square roughness, larger than the monolayer chain length of 1-2 nm. Hence, unlike SPM experiments and MD simulations, asperities go in and out of contact ("rejuvenate") during sliding, and have a well-defined average contact time at constant velocity.

\section{Rate-state theory}

The time and velocity dependencies of friction have been studied in macroscale systems in detail [2, 20-23]. Various models, such as the Ruina-Dieterich slip law for dry systems $[\underline{2} \underline{23}]$, have been proposed to capture a variable friction coefficient. This law takes the form 


$$
\mu(\phi, \dot{x})=\mu_{0}+A \ln \left(1+\frac{|\dot{x}|}{v_{c}}\right)+B \ln \left(1+\frac{\phi}{\phi_{c}}\right)
$$

In this equation, $\dot{x}$ is the instantaneous block velocity, and $\phi(s)$ is the contact age that represents the effective static aging time. $A$ and $B$ are positive coefficients that tune the strength of the rate and state effects, respectively. Parameters $v_{c}$ and $\phi_{c}$ nondimensionalize velocity and time. Unity has been added to the logarithms to prevent divergence such that $\mu_{0}$ is the minimum friction coefficient. A coupled equation, such as the Dietrich-Ruina aging law, is needed to describe how $\phi$ changes with time:

$$
\dot{\phi}=1-\frac{\phi|\dot{x}|}{d_{c}}
$$

In this model, $d_{c}$ is the characteristic memory length. At steady sliding, $\phi_{s s}=d_{c} / v_{p}$, where $\dot{x}=v=v_{p}$ has been assumed ( $v_{p}$ is the puller velocity). For steady sliding, the contact age exponentially approaches or decays to this steady-state value depending on initial conditions. For a surfaces at rest, $\dot{\phi}=1$, and $\mu$ scales logarithmically with time.

Of importance here is the behavior of this model due to a jump in puller velocity from $v_{p 1}$ to $v_{p 2}$. We can identify three key times. At $t_{1}$ as in Fig. 1, we can assume steady state pulling at $\dot{x}=v_{p 1}$ such that $\phi_{1}=d_{c} / v_{p 1}$. At a time $t^{*}$ just after the jump in velocity, the instantaneous velocity is now $\dot{x}=v_{p 2}$ but the state has not yet had time to evolve such that the contact age remains $\phi^{*}=d_{c} / v_{p 1}$. At $t_{2}$, corresponding to a long time after the jump (for practical purposes when the block has moved several times $d_{c}$ ), the state $\phi_{2}=d_{c} / v_{p 2}$. We can insert these values into Eq. (1) to calculate changes in friction assuming unity added in the logarithms is insignificant. The height of the friction spike is then $\mu\left(\phi^{*}, v_{p 2}\right)-\mu\left(\phi_{1}, v_{p 1}\right)=A \ln \left(v_{2} / v_{1}\right)$. Similarly, the change in steady state value $\mu\left(\phi_{2}, v_{p 2}\right)-\mu\left(\phi_{1}, v_{p 1}\right)=(A-B) \ln \left(v_{p 2} / v_{p 1}\right)$. These quantities are labeled in Fig. 1. Friction spike behavior for $\mu \mathrm{m}$-scale asperities between macroscale objects has been observed in a wide variety of interfaces, including self-mated soda-lime glass [ㅍ], wood, rock [무 $\underline{24}]$, cardboard [마], and also for Teflon and steel $[\underline{3}, \underline{25}]$.

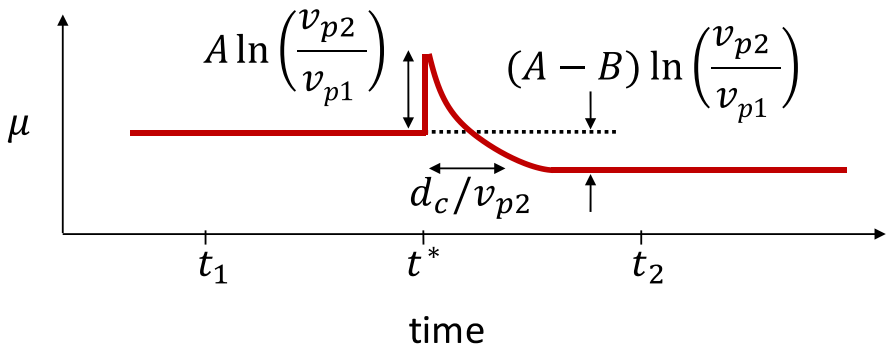

Fig. 1: Analytical response of the friction coefficient to a velocity jump assuming $v_{p 2}>v_{p 1}$. If the reverse is true, the friction spike points downward.

For a massless system, the spring elongation $e$ will simply be proportional to $\mu$. In the system described below, the mass is small, but not small enough for the inertia to be ignored. A full rate-state simulation is needed to extract the coefficients $A, B$ and $d_{c}$ from elongation versus time curves. This involves numerically integrating the block's equation of motion and Dietrich-Ruina aging law. 


\section{3. Experimental setup and methods}

\subsection{Device overview}

To unravel the true contribution to $\mu$ from $v$ under steady sliding conditions in a representative micromachined multi-contact interface with nanometer-scale asperities, we have devised an on-chip friction platform with a constant-velocity actuator [26]. This device is capable of the velocity jump shown in Fig. 1. The device is processed by a standard micromachining process flow [27], and hence satisfies the characteristics described in the last paragraph of the introduction. Devices were coated by vapor deposition with a perfluorinated monolayer tridecafluorotris(dimethylamino)silane [FOTAS,

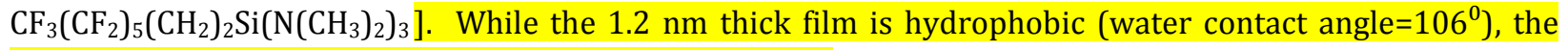
chains in this boundary lubricant are likely disordered [ $\underline{6}]$.

The experimental platform is schematically represented in Fig. 2a, with key components comprising a block of mass $m_{b}$ subject to a normal load $N_{b}$, a spring of stiffness $k$, and an actuator that pulls at constant velocity $v_{p}$. An ideal setup to measure frictional properties will implement this schematic and enable wide variation of these parameters (e.g. [이]). Previous MEMS test platforms (with comb drive $[\underline{28}, \underline{29}]$ or stepper motor [으] actuators) do not achieve the vital constant velocity functionality. By injecting heat via Joule heating at a variable rate into the thermal actuator legs, the new platform enables constant velocity and a wide variation of $v_{p}(1-3100 \mu \mathrm{m} / \mathrm{s}$ with $<5 \%$ variation in velocity). It does this while providing a large (at this scale) pulling force of tens of $\mathrm{mN}$, much greater than the friction force, and hence the $v_{p}$ value is not influenced by the frictional load. An open-loop, iterative method is used to tune voltage signals to achieve arbitrary velocity profiles [26], including constant $v_{p}$ and an instantaneous velocity jump. 

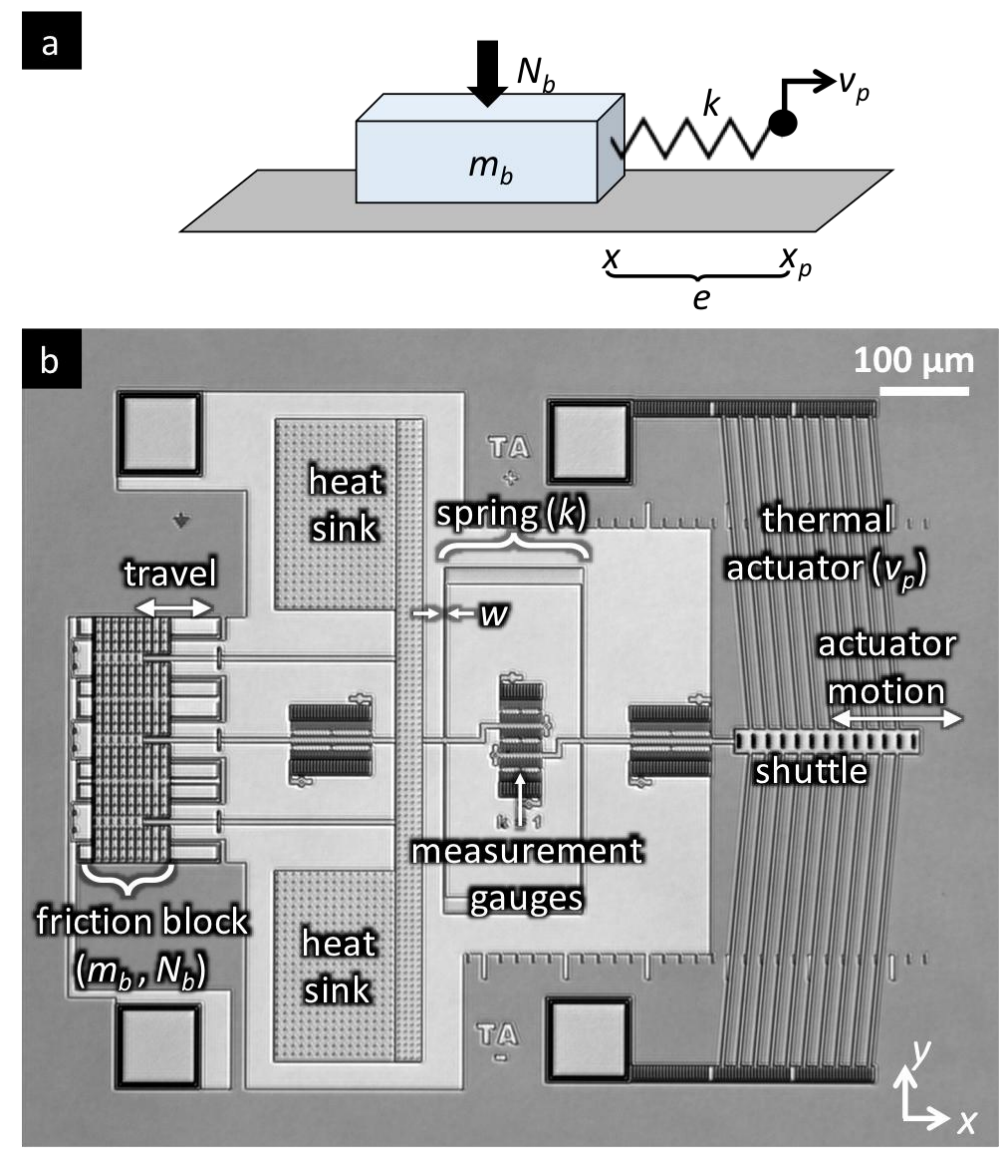

Fig. 2: (a) Schematic of a puller/spring/block system. (b) Optical image of a fabricated, released and monolayer-coated device.

\subsection{Frictional interface}

As seen in Fig. 3a, the friction block comprises a large area $\left(\sim 14,000 \mu \mathrm{m}^{2}\right)$ over which electrostatic loading is applied and a smaller area $\left(\sim 1000 \mu \mathrm{m}^{2}\right)$ in which there are six frictional feet in contact with a substrate. Electrostatic loading was used apply normal load $N_{b}$ with voltage $V_{b}$ using an electrode under the plate area. The counterfaces of the feet are each electrically grounded such that there is no electric field across the monolayer lubricant in the rubbing interface (where the friction feet make contact with the bottom countersurface). Three beams connect the block to the spring near the middle of the block. The block thereby can act to resist the net moment applied by the beams, which will minimize any potential tilting effects. Using interferometry, no evidence of tiling was found even when the beams were connected to the end of the block as long as a small voltage $V_{b}$ was applied [30]. Hence the friction feet are in contact with the bottom countersurface over their entire length. 

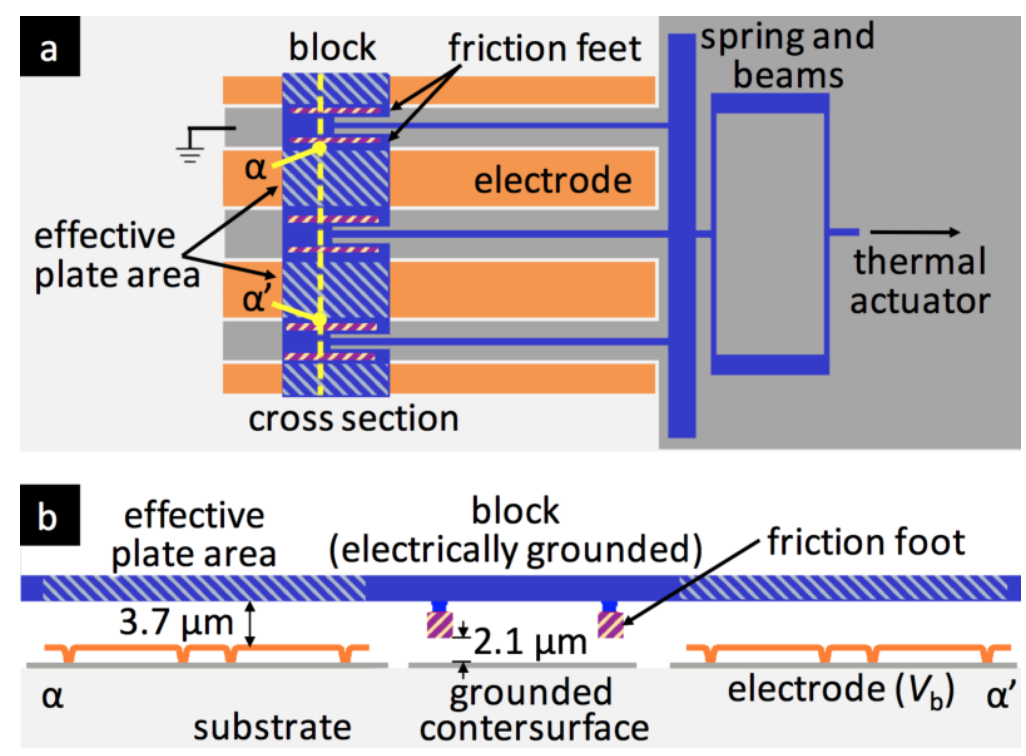

Fig. 3: (a) Top and (b) cross section (through the yellow dotted line $\left.\alpha \alpha^{\prime}\right)$ schematic views of the block. Contact between the block and substrate occurs at six friction feet (hashed purple). The four effective parallel plates (orange) apply the normal load to the block via electrostatic loading. $\mathrm{A} \sim 1 \mathrm{~nm}$ thick monolayer lubricant (not represented) has been applied to all surfaces.

\subsection{Displacement extraction}

Displacements were extracted from images taken by an optical microscope using a 50X long working distance objective $(N A=0.42)$. Measurement gauges (see Fig. $2 \mathrm{~b}$ for optical image and Fig. 4 for a schematic) form a periodic waveform (pixel intensity $I(x)$ ) to which a Fourier series was fit. Changes in the phase of this waveform in subsequent images were converted to a displacement. The displacement resolution using this technique is $1.2 \mathrm{~nm}$.

A Phantom v1211 high-speed camera (5 $\mu$ s temporal resolution) captured video, which was saved and postprocessed. A SugarCUBE Quad LED light source provided illumination. The exposure time was maintained at $5 \mu \mathrm{s}$ for all images in order to minimize blur. A frame was taken every $100 \mathrm{~nm}$ of travel, which corresponds to 30,000 frames per second (fps) at $v_{p}=3000 \mu \mathrm{m} / \mathrm{s}$. The total travel distance was $10 \mu \mathrm{m}$, so that in a single run, we obtain 100 measurements. Each point in Fig. 5 below is an average of 5 runs. The maximum frame rate of 200,000 fps was used in the velocity jump test, as described below. 


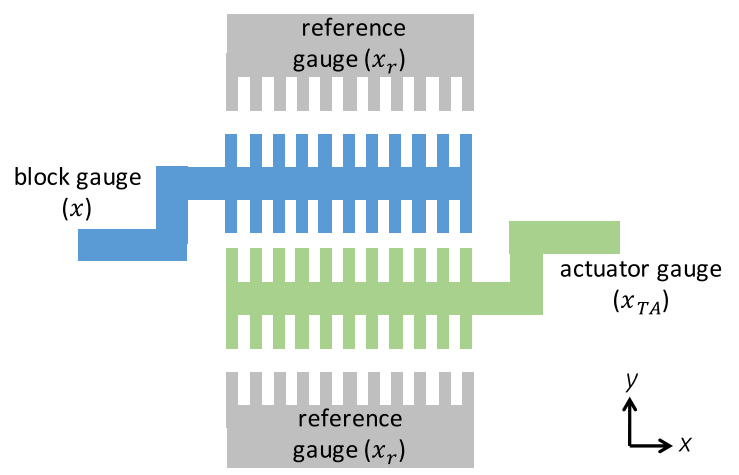

Fig. 4: Schematic of the measurement gauges located in the center of the fixed-guided beams.

\section{4. Results}

139 In Fig. 5, we present the effect of increasing $v_{p}$ on $\mu$. The data is taken in the steady sliding regime with high $k$ $140(100 \mathrm{~N} / \mathrm{m})$ (stick-slip behavior occurs at higher $N_{b}$ or lower $\left.k\right)$. The friction coefficient is defined as $141 \mu \equiv k \bar{e} / N_{b}$, where $\bar{e}$ is the average spring elongation. From 0.1 to $\sim 300 \mu \mathrm{m} / \mathrm{s}, \mu$ falls with increasing $v_{p}$, 142 which scales with the decrease in time that asperities remain in contact. At higher $v_{p}, \mu$ increases again. This 143 increase indicates velocity strengthening and can be attributed to the increasing contribution of interfacial 144 viscous effects.

145 The solid black line in Fig. 5 represents a theoretical fit using the model given in Eqs. (1) and (2) with $146 A=0.013, B=0.024, \mu_{0}=0.19, d_{c}=4.3 \mathrm{~nm}, v_{c}=10 \mu \mathrm{m} / \mathrm{s}$, and $\phi_{c}=10 \mu \mathrm{s}$. These are based on measured 147 values from tens of devices to be reported elsewhere. Although a $R^{2}$ value is not appropriate for nonlinear 148 fits, the definition $R^{2} \equiv 1-S S_{\text {res }} / S S_{\text {tot }}$ where $S S_{\text {res }}$ is the residual sum of squares and $S S_{\text {tot }}$ is the total sum 149 of squares can be used to gain a sense of the magnitude of the residuals [31]. This results in $R^{2}=0.54$ using 150 the data from the three devices. However, this is not a best fit for a single device. For this mean fit, a value of $151 R^{2}=0.71$ using device 1 alone. This reasonably good fit would be further improved if the above parameters 152 were optimized for this device alone. 


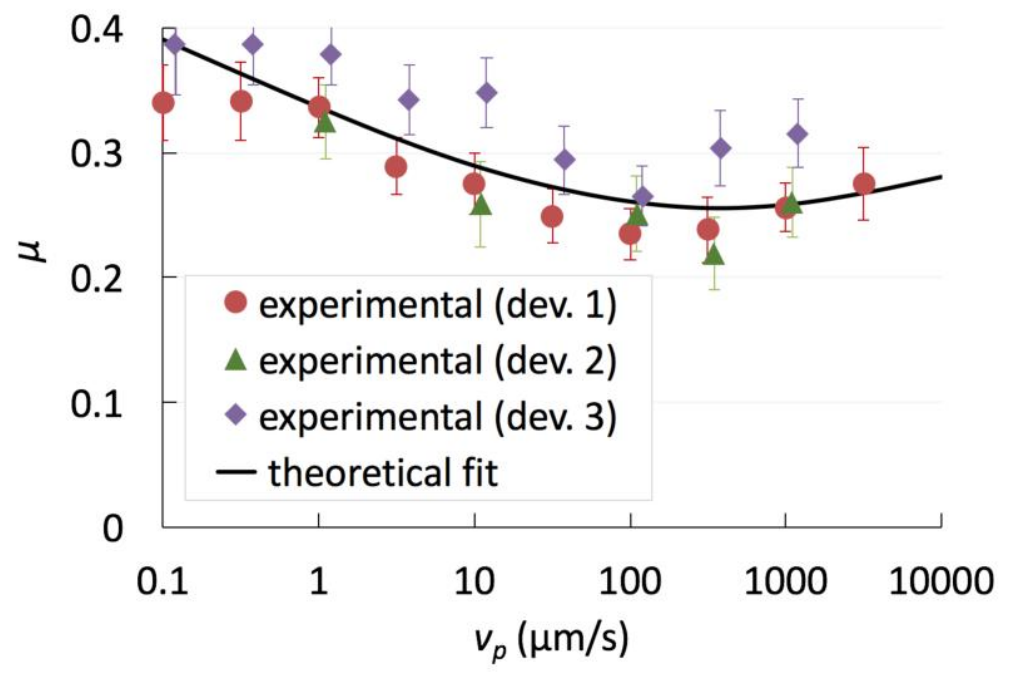

Fig. 5: Dynamic friction coefficient $\mu$ vs. actuator pulling velocity $v_{p}$ in the steady-sliding regime $\left(\dot{x}=v_{p}\right)$. Three devices are shown. Data from devices 2 and 3 are offset horizontally by $0.1 \cdot v_{p}$ and $0.2 \cdot v_{p}$, respectively (the offset is a multiple of $v_{p}$ due to the $\log$ scale). Here $k=100 \mathrm{~N} / \mathrm{m}$ and $N_{b}=14.12 \mu \mathrm{N}$. Error bars represent one standard deviation of $\mu$ by considering the timeweighted variation in spring elongation of multiple trials.

There is a clear trend in decreasing $\mu$ over three orders of magnitude of increasing $v_{p}$. The minimum in $\mu$ only occurs near the maximum velocity in our experimental range. While consistently observed, the subsequent increase in $\mu$ is small. An independent method to observe velocity strengthening involves observing the transient friction response in a velocity jump test [ $\underline{3}$ ]. As described above, the velocity must change over a distance much less than the asperity contact diameter. In the present experiments, the jump takes places in $\sim 0.2 \mu \mathrm{s}$ as limited by the slew rate in our amplifiers. Over this time, the block moves $\approx(1000 / 2) \mu \mathrm{m} / \mathrm{s} \cdot 0.2 \mu \mathrm{s}=0.1 \mathrm{~nm}$. Fig. 6a shows the jump in actuator velocity from $50 \mu \mathrm{m} / \mathrm{s}$ to $1000 \mu \mathrm{m} / \mathrm{s}$ at $t^{*}=3 \mathrm{~ms}$. Fig. $6 \mathrm{~b}$ shows the block response to this jump. At $t^{*}$, a friction spike is observed (see inset). The high temporal and spatial resolution of the experimental setup enables observation of these spikes. To cancel some of the measurement noise and surface variation, an average of the trials is shown in black. Before the velocity jump, $e(t) \approx 42 \pm 0.4$ (one standard deviation) $\mathrm{nm}$ in steady state shows some variation due to local differences in friction. At the jump, $e$ spikes and then decays over a characteristic distance $d_{c}$, and finally decays to a new steady-state value $e(t) \approx 40 \pm 0.4 \mathrm{~nm}$. 


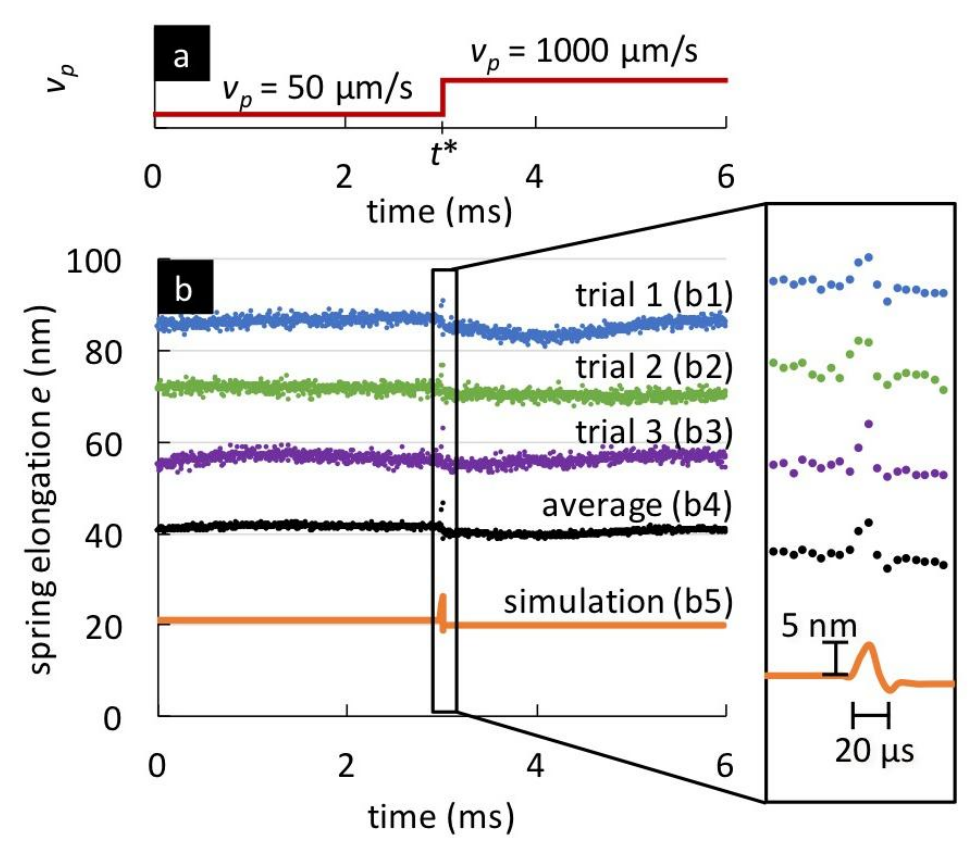

Fig. 6: (a) Pulling velocity profile. (b1,b2,b3) Spring elongation vs. time for three runs of the same device, offset by $+15,+30$, and +45 $\mathrm{nm}$ with respect to the average in (b4). The camera framerate was 200,000 fps. Here, $k=100 \mathrm{~N} / \mathrm{m}$ and $N_{b}=15.42 \mu \mathrm{N}$. (b4) Average over 5 runs of the same device where $e$ is the actual elongation. (b5) Simulation results offset by $-20 \mathrm{~nm}$.

The positive spike can be attributed to the aforementioned interfacial viscous effect in which $\mu$ increases with the instantaneous increase in block velocity $\Delta \dot{x}$. The subsequent decrease in the steady state value of $e$ is the result of a combination of the loss in aging time of the contacts and this direct effect. In Fig. 6b, a numerical simulation (labeled "b5") is shown calculated by integrating Eqs. (1) and (2) with time. This velocity jump test provides estimates of the velocity strengthening coefficient $A$, the memory coefficient $B$ and the value of $d_{c}$ used in rate-state modeling. The predicted steady-sliding values of $\mu$ as a function of $v_{p}$ are shown in Fig. 5 (black solid line).

\section{Discussion and conclusions}

The numerical model we have implemented using rate-state equations is phenomenological in nature. Nevertheless, we can gain useful physical insight into the factors that control friction. Earlier results indicate there is indeed static friction aging $[\underline{6}, \underline{30}]$. The decrease in friction with velocity up to $300 \mu \mathrm{m} / \mathrm{s}$ suggests that $\phi=d_{c} / v_{p}$ is the dominant factor. From MD simulations, the increase in friction with velocity for $v_{p}>300 \mu \mathrm{m} / \mathrm{s}$ may saturate at extremely high $v_{p}$ beyond $0.2 \mathrm{~m} / \mathrm{s}$ (speeds not obtainable here) []].

Another important point of discussion is regarding the value of the memory length, $d_{c} \approx 4 \mathrm{~nm}$, that we extracted using the full rate-state model. First, we now see that the memory length concept extends from the micrometer $[\underline{3}, \underline{20}]$ to the nanometer scale. The contact diameter is expected to be $\sim 13 \mathrm{~nm}$ (calculated using a Greenwood-Williamson model [32]), while the FOTAS chain length is $\sim 1 \mathrm{~nm}$. Second, the $d_{c}$ value may be related to the quality (strength of the chemical bonds) or quantity (the real contact area) of the contacts. For an $\mathrm{SiO}_{2} / \mathrm{SiO}_{2}$ interface, chemical bonding may be the main mechanism for aging, implying a memory length on 
the order of the bond length [33], corresponding to the quality of the contacts. Alternatively, in dry multicontact systems, $d_{c}$ is unambiguously associated with the asperity diameter $[\underline{3}, \underline{20}]$, corresponding to the quantity of contacts with the inherent assumption that chemical bonding is instantaneous. Indeed, the ratestate theory we have implemented is based on experiments where only the real area of contact affects the state. A single-valued $d_{c}$ represents an effective value extracted from modeling, and the fact that it is intermediate to the chain length and contact diameter suggests that both play a role in determining its value. Two length scales may play a role here. From MD simulations, the monolayer chains compress, tilt, and displace to accommodate advancing asperities [34]. This will occur over a $\sim 1 \mathrm{~nm}$ length. The chains then become confined between asperities, and may continue to reconfigure over the length of the contact diameter ("shear-induced ordering") [ㅁ5]

In conclusion, models and experiments indicate that single asperity monolayer friction increases with or is independent of velocity. We have shown that friction in a micromachined interface consisting of nanometerscale asperities coated by FOTAS decreases with velocity from $\sim 0.1$ to $\sim 300 \mu \mathrm{m} / \mathrm{s}$ and attribute this to the decreasing asperity lifetime. This important effect will only manifest in a multi-asperity test as we have conducted here. At $\sim 300 \mu \mathrm{m} / \mathrm{s}$, friction begins to increase. This component of friction was confirmed in a velocity jump test. The present results reflect a fluorine-terminated monolayer lubricant. Multi-asperity friction results for methyl-terminated lubricants, as most often studied in MD simulations and SPM experiments, may differ. Therefore, further research is needed to better understand the relationship between single and multi-asperity monolayer-coated interfaces.

Measurement of rate and state frictional properties is useful for a variety of reasons. For example, it should be possible to predict the stick-slip to steady sliding bifurcation curve $[\underline{2}, \underline{22}, \underline{23}]$, a stability related phenomenon, for arbitrary combinations of $N_{b}, k$, and $v_{p}$. Stick-slip behavior can cause damage to interfaces, as large amounts of energy are released in short times during the slip phase [36]. To minimize energy dissipation and enable precision placement, the sliding regime can be guaranteed. Finally, feed-forward control can be used effectively when plant characteristics, including frictional properties, are well defined $[\underline{37}, \underline{38}]$.

\section{Acknowledgements}

This work was supported by the National Science Foundation (NSF) under Grant CMMI 1030322. The work of S.S. was supported by the NSF Graduate Research Fellowship Program. The authors thank the staff of Sandia National Laboratories Microelectronics Development Laboratory for the fabrication of the devices used in these experiments.

\section{References}

[1] F. P. Bowden and D. Tabor, The friction and lubrication of solids vol. 2: Wiley Online Library, 1964.

[2] A. Ruina, "Slip instability and state variable friction laws," J. of Geophysical Research, vol. 88, pp. 10359-10370, 1983.

[3] J. H. Dieterich and B. D. Kilgore, "Direct observation of frictional contacts: New insights for statedependent properties," Pure and Applied Geophysics, vol. 143, pp. 283-302, 1994.

[4] R. Maboudian and C. Carraro, "Surface chemistry and tribology of MEMS," Annu. Rev. Phys. Chem., vol. 55, pp. 35-54, 2004. 
[5] S. H. Kim, D. B. Asay, and M. T. Dugger, "Nanotribology and MEMS," Nano today, vol. 2, pp. 22-29, 2007.

[6] A. D. Corwin and M. P. de Boer, "Frictional aging and sliding bifurcation in monolayer-coated micromachines," J. Microelectromech. Sys, vol. 18, pp. 250-262, 2009.

[7] P. T. Mikulski and J. A. Harrison, "Packing-density effects on the friction of n-alkane monolayers," Journal of the American Chemical Society, vol. 123, pp. 6873-6881 2001.

[8] N. J. Brewer, B. D. Beake, and G. J. Leggett, "Friction force microscopy of self-assembled monolayers: influence of adsorbate alkyl chain length, terminal group chemistry, and scan velocity," Langmuir, vol. 17, pp. 1970-1974, 2001.

[9] J. Kiely and J. Houston, "Contact hysteresis and friction of alkanethiol self-assembled monolayers on gold," Langmuir, vol. 15, pp. 4513-4519, 1999.

[10] L. Prandtl, "Ein Gedankenmodell zur kinetischen Theorie der festen Körper," ZAMM - Journal of Applied Mathematics and Mechanics/Zeitschrift für Angewandte Mathematik und Mechanik, vol. 8, pp. 85-106, 1928.

[11] M. H. Müser, "Velocity dependence of kinetic friction in the Prandtl-Tomlinson model," Physical Review B, vol. 84, p. 125419, 2011.

[12] E. Riedo, E. Gnecco, R. Bennewitz, E. Meyer, and H. Brune, "Interaction potential and hopping dynamics governing sliding friction," Physical review letters, vol. 91, p. 084502, 2003.

[13] Q. Li, Y. Dong, D. Perez, A. Martini, and R. W. Carpick, "Speed dependence of atomic stick-slip friction in optimally matched experiments and molecular dynamics simulations," Physical review letters, vol. 106, p. 126101, 2011.

[14] M. Chandross, C. D. Lorenz, M. J. Stevens, and G. S. Grest, "Simulations of nanotribology with realistic probe tip models," Langmuir, vol. 24, pp. 1240-1246, 2008.

[15] R. L. Jones, N. C. Pearsall, and J. D. Batteas, "Disorder in alkylsilane monolayers assembled on surfaces with nanoscopic curvature," The Journal of Physical Chemistry C, vol. 113, pp. 4507-4514, 2009.

[16] X. Xiao, J. Hu, D. H. Charych, and M. Salmeron, "Chain length dependence of the frictional properties of alkylsilane molecules self-assembled on mica studied by atomic force microscopy," Langmuir, vol. 12, pp. 235-237, 1996.

[17] A. Lio, D. Charych, and M. Salmeron, "Comparative atomic force microscopy study of the chain length dependence of frictional properties of alkanethiols on gold and alkylsilanes on mica," The Journal of Physical Chemistry B, vol. 101, pp. 3800-3805, 1997.

[18] S. Lee, Y.-S. Shon, R. Colorado, R. L. Guenard, T. R. Lee, and S. S. Perry, "The influence of packing densities and surface order on the frictional properties of alkanethiol self-assembled monolayers (SAMs) on gold: a comparison of SAMs derived from normal and spiroalkanedithiols," Langmuir, vol. 16, pp. 2220-2224, 2000.

[19] B. G. Bush, F. W. Del Rio, C. Jaye, D. A. Fischer, and R. F. Cook, "Interfacial Mechanical Properties ofAlkylsilane Monolayers on Silicon Substrates," Microelectromechanical Systems, Journal of, vol. 22, pp. 34-43, 2013.

[20] F. Heslot, T. Baumberger, B. Perrin, B. Caroli, and C. Caroli, "Creep, stick-slip, and dry-friction dynamics: Experiments and a heuristic model," Physical Review E, vol. 49, p. 4973, 1994.

[21] G. Perrin, J. R. Rice, and G. Zheng, "Self-healing slip pulse on a frictional surface," J. of the Mechanics and Physics of Solids, vol. 43, pp. 1461-1495, 1995.

[22] J.-C. Gu, J. R. Rice, A. L. Ruina, and S. T. Tse, "Slip motion and stability of a single degree of freedom elastic system with rate and state dependent friction," J. of the Mechanics and Physics of Solids, vol. 32, pp. 167-196, 1984. 
[23] A. Ruina, "Stability of steady frictional slipping," J. of Applied Mechanics, vol. 50, pp. 343-349, 1983.

[24] J. H. Dieterich, "Modeling of rock friction: 1. Experimental results and constitutive equations," J. of Geophysical Research, vol. 84, pp. 2161-2168, 1979.

[25] P. E. Dupont and E. P. Dunlap, "Friction modeling and control in boundary lubrication," 1993, pp. 1910-1915.

[26] S. S. Shroff and M. P. de Boer, "Constant Velocity High Force Microactuator for Stick-Slip Testing of Micromachined Interfaces," J. Microelectromech. Sys (available online), 2015.

[27] J. J. Sniegowski and M. P. de Boer, "IC-compatible polysilicon surface micromachining," Annual Review of Materials Science, vol. 30, pp. 299-333, 2000.

[28] D. H. Alsem, M. T. Dugger, E. A. Stach, and R. O. Ritchie, "Micron-scale friction and sliding wear of polycrystalline silicon thin structural films in ambient air," J. Microelectromech. Sys, vol. 17, pp. 11441154, 2008.

[29] W. M. van Spengen and J. W. Frenken, "The Leiden MEMS tribometer: Real time dynamic friction loop measurements with an on-chip tribometer," Tribology letters, vol. 28, pp. 149-156, 2007.

[30] S. S. Shroff, N. Ansari, W. R. Ashurst, and M. P. de Boer, "Rate-state friction in microelectromechanical systems interfaces: Experiment and theory," Journal of Applied Physics, vol. 116, p. 244902, 2014.

[31] G. A. Seber and A. J. Lee, Linear regression analysis vol. 936: John Wiley \& Sons, 2012.

[32] J. Greenwood and J. Williamson, "Contact of nominally flat surfaces," Proceedings of the Royal Society of London. Series A. Mathematical and Physical Sciences, vol. 295, pp. 300-319, 1966.

[33] Q. Li, T. E. Tullis, D. Goldsby, and R. W. Carpick, "Frictional ageing from interfacial bonding and the origins of rate and state friction," Nature, vol. 480, pp. 233-236, 2011.

[34] J. A. Harrison, J. D. Schall, M. T. Knippenberg, G. Gao, and P. T. Mikulski, "Elucidating atomic-scale friction using molecular dynamics and specialized analysis techniques," Journal of Physics: Condensed Matter, vol. 20, p. 354009, 2008.

[35] B. Bhushan, J. N. Israelachvili, and U. Landman, "Nanotribology: friction, wear and lubrication at the atomic scale," Nature, vol. 374, pp. 607-616, 1995.

[36] A. D. Berman, W. A. Ducker, and J. N. Israelachvili, "Origin and characterization of different stick-slip friction mechanisms," Langmuir, vol. 12, pp. 4559-4563, 1996.

[37] S. C. Southward, C. J. Radcliffe, and C. MacCluer, "Robust nonlinear stick-slip friction compensation," Journal of Dynamic Systems, Measurement, and Control, vol. 113, pp. 639-645, 1991.

[38] B. Armstrong-Hélouvry, P. Dupont, and C. C. De Wit, "A survey of models, analysis tools and compensation methods for the control of machines with friction," Automatica, vol. 30, pp. 1083-1138, 1994. 